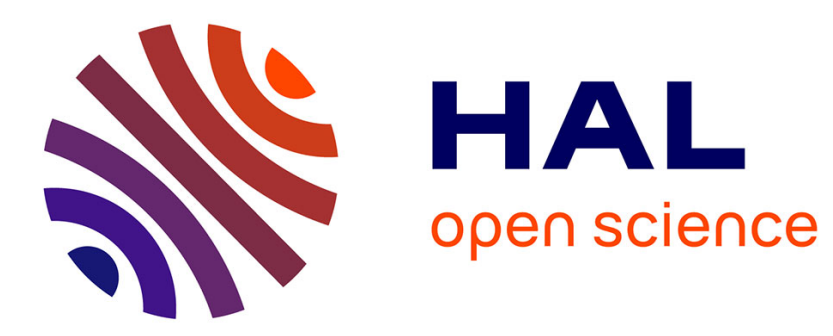

\title{
Advancing liquid contact line on visco-elastic gel substrates: stick-slip vs. continuous motions
}

Tadashi Kajiya, Adrian Daerr, Tetsuharu Narita, Laurent Royon, François

Lequeux, Laurent Limat

\section{- To cite this version:}

Tadashi Kajiya, Adrian Daerr, Tetsuharu Narita, Laurent Royon, François Lequeux, et al.. Advancing liquid contact line on visco-elastic gel substrates: stick-slip vs. continuous motions. Soft Matter, 2013, 9 (2), pp.454-461. 10.1039/C2SM26714D . hal-02367071

\section{HAL Id: hal-02367071 https://hal.science/hal-02367071}

Submitted on 17 Nov 2019

HAL is a multi-disciplinary open access archive for the deposit and dissemination of scientific research documents, whether they are published or not. The documents may come from teaching and research institutions in France or abroad, or from public or private research centers.
L'archive ouverte pluridisciplinaire $\mathbf{H A L}$, est destinée au dépôt et à la diffusion de documents scientifiques de niveau recherche, publiés ou non, émanant des établissements d'enseignement et de recherche français ou étrangers, des laboratoires publics ou privés. 


\title{
Advancing Liquid Contact Line on Visco-Elastic Gel Substrates: Stick-Slip vs Continuous Motions
}

\author{
Tadashi Kajiya ${ }^{(a) *}$, Adrian Daerr ${ }^{(a)}$, Tetsuharu Narita ${ }^{(b)}$, \\ Laurent Royon ${ }^{(a)}$, François Lequeux ${ }^{(b)}$ and Laurent Limat ${ }^{(a)}$ \\ (a) Laboratoire MSC, UMR 7057, CNRS, \\ Université Paris Diderot, Bâtiment Condorcet, \\ 10 rue Alice Domon et Léonie Duquet, 75205 Paris Cedex 13, France \\ (b) PPMD-SIMM, UMR 7615, CNRS, UPMC, ESPCIParisTech, \\ 10 rue Vauquelin, 75231 Paris Cedex 05, France
}

(Dated: September 28, 2012)

\begin{abstract}
We studied the dynamics of water sessile droplets advancing on hydrophobic and visco-elastic Poly(styrene-butadiene-styrene)(SBS)-paraffin gel substrates at various inflation rates. During the advancing process, the droplet contact line exhibits three different regimes of motions. When the contact line advances at a high velocity, the contact line moves continuously with a constant contact angle. As the contact line slows down, it starts stick-slip motion: the contact line is pinned at a certain position, then suddenly slips forward. With further decrease of the velocity, the contact line stops stick-slip and continuously advances again. The observed threshold values for the transitions of the contact line motions (continuous - stick-slip - continuous) indicate that the rheology of the gel drastically affects the dynamics of liquid on its surface. We suggest that on visco-elastic gels, the moving contact line exhibits both aspects of wetting on elastic solids and wetting on viscous liquids depending on the characteristic frequency of the gel surface deformation. At an intermediate regime, the stick-slip motion of the contact line appears. We also propose a simple geometrical model in the stick-slip regime which allows us to relate the jumps of droplet radius to the jumps of apparent contact angle.
\end{abstract}

\footnotetext{
* Current affiliation is PPMD-SIMM, UMR 7615, CNRS, UPMC, ESPCIParisTech, 10 rue Vauquelin, 75231 Paris Cedex 05, France
} 


\section{INTRODUCTION}

Gels, cross-linked network of polymers swollen with solvent, are materials which have been attracting continued interest for decades since they are complex nature of matter $[1,2]$ and they also have promising potentials for applications such as food processing, medical science [3], and organic electronics [4]. One of the important challenges is to understand and to control the interfacial properties of gels, as they determine adhesion and friction [5-7] (e.g. cartilage replacement), surface tension and wetting properties [8-11] (e.g. soft contact lens, anti-condensation coating, encapsulation in consumer products). Here we are focusing on the wetting of liquids on gel surfaces.

Wetting dynamics is still an active subject of research even on "hard" solid surfaces because of the need to patch-up classical hydrodynamics near the contact line $[13,14]$. On gels, the situation would be more complex as the wetting liquid can cause a large deformation on the gel surface which successively affects the statics and dynamics of the contact line [12]. The deformation of the gel surface is caused by two mechanisms: by the balance between the interfacial tensions and elastic resistance of gel and by the volume exchange between the liquid and gel. Intuitively, the behavior of the contact line on gels might be understood in analogy with the wetting on soft surfaces like elastomer [15-17] or with the wetting on permeable surfaces like porous media $[18,19]$. However, as gels have unusual natures between solids and liquids, the wetting on gels would even be more complex than those situations. Previously, Holmes et al. [20] and Kajiya et al. [21] studied the spreading behavior of liquid droplets on gels which are under the swelling equilibrium, and they observed a strong coupling between the volume exchange and contact line dynamics.

In this paper, we are turning our attention to a different aspect of the gel complexity, i.e., the rheology of gels. We studied the dynamics of water sessile droplets being inflated on poly(styrene-butadiene-styrene)(SBS) - paraffin gel substrates. As SBS-paraffin gel is hydrophobic and there is no volume exchange between the droplet and gel, the possible factor causing the surface deformation is the interfacial tensions applied at the contact line. Nevertheless, unlike the case of wetting on elastomer, the droplet contact line exhibits quite complex behaviors, i.e., the contact line shows two regimes of continuous advancing motion, and a stick-slip motion depending on the inflation rate and on droplet radius. The stick-slip motion of the contact line was previously observed by $\mathrm{Pu}$ et al. in the situation of wetting 
on thin polymer films $[22,23]$. Here we found that on a SBS-Paraffin gel, there are three different regimes of the contact line motion, i.e., continuous, stick-slip, and another continuous motion. We discuss how the transitions of these contact line motions are characterized by the parameters such as the frequency of the surface deformation and the gel rheology, and propose a possible mechanism for the present phenomena.

\section{EXPERIMENTAL SECTION}

Poly(styrene-butadiene-styrene)(SBS) - paraffin gels were used for the gel substrates and distilled water (Milli-Q Integral; Millipore, USA) was used for the liquid.

SBS powders (G1682; Kraton Polymers, USA) were dissolved in paraffin (Norpar15; ExxonMobil; USA) heated in a water bath at $90^{\circ} \mathrm{C}$. After the SBS was completely dissolved in paraffin, the solution was poured into a gel mold and was cooled down to ambient temperature. The gel mold consists of two glass plates separated by a rubber spacer. The gel samples were $70 \mathrm{~mm}$ of length, $20 \mathrm{~mm}$ of width and $3 \mathrm{~mm}$ of thickness. Gels of various SBS concentrations were prepared. The mass concentration of SBS $c_{S B S}$ was varied from $8 \%$ to $25 \%$. The typical roughness of the gel surface was measured to be of order $10 \mathrm{~nm}$ by an optical profiler.

The rheological property of the gel was measured by a strain-controlled rheometer (Physica MCR 500; Anton Paar, Austria) with a stainless steel cone and plate sample cell. The radius of the plate is $50 \mathrm{~mm}$, cone angle is $1^{\circ}$, and the gap between the cone and plate was set to $0.052 \mathrm{~mm}$. A shear strain amplitude was set to $1 \%$. A strain sweep test for selected samples showed that this was sufficiently small to keep rheological properties in the linear viscoelastic range. The experiments were conducted at $20 \pm 0.2^{\circ} \mathrm{C}$ and performed at frequencies from $10^{-4} \mathrm{~Hz}$ to $10 \mathrm{~Hz}$.

The curve of storage modulus $G$ ' and loss modulus $G$ " against frequency are plotted in fig. 1 for the gel of $c_{S B S}=10 \%$, and typical values of the gel properties $\left(G^{\prime}\right.$ at $f=1 \mathrm{~Hz}$ and crossover frequency $f_{\text {cross }}$ ) are listed in tab. I for $c_{S B S}=8,10,12,16 \%$. Note that two types of measurements were conducted: one at low frequencies $\left(10^{-4}-10^{-2} \mathrm{~Hz}\right)$ to determine $f_{\text {cross }}$, and the other at a wide range of frequencies $\left(10^{-4}-10 \mathrm{~Hz}\right)$ to determine $G^{\prime}$ at $f=1$ Hz. For $c_{S B S}=12 \%$ and $16 \%, f_{\text {cross }}$ was obtained by extrapolating the curves of $G$ ' and G". 
The inflation experiment of a water droplet on the gel was conducted as a following procedure. A water droplet was placed on a gel by a micro-syringe. The syringe was connected to a motor syringe pump (Model 33; Harvard Apparatus; USA), and it supplies water to the droplet and inflate it at a constant volume rate $q$. The inflation rate $q$ was varied from $1 \mu \mathrm{l} / \mathrm{min}$ to $200 \mu \mathrm{l} / \mathrm{min}$. From the side and top of the droplet, the shape of the droplet was monitored by 2 CCD cameras (Model A101fc and Model PLA1000; Basler; Germany) with magnification lenses (CCTV lens; Pentax, Japan).

Alternatively, in the situation of a static contact line, the surface profile of the gel was measured by an optical profiler (Microsurf 3D, Fogal Nanotech, France) with a $5 \times$ objective lens. A sessile droplet was placed on a SBS-paraffin gel. Successively, the gel surface profile close to the contact line was measured at different time steps.

\section{EXPERIMENTAL RESULTS}

\section{A. Stick-slip motion of contact line}

Figure 2 (a)-(d) shows sequential pictures of a water droplet being inflated on a SBSparaffin gel of $c_{S B S}=10 \%$ at an inflation rate $q=20 \mu \mathrm{l} / \mathrm{min}$. During the inflation process, the droplet contact line exhibits a stick-slip motion. The contact line is pinned at a certain position. As the inflation proceeds, the contact angle gradually increases while the contact line stays at the same position. Then suddenly, the contact line slips forward over a certain distance. Successively, the contact line repeats this stick-slip motion.

After the inflation process, the water droplet was removed quickly and a picture of the gel surface was taken as it is shown in fig. 2 (e). Multiple circular stripe patterns are observed on the gel surface. These circular patterns indicate that the droplet contact line locally pulls up the gel surface, forming ridges. After a sufficiently long time, i.e., $2-3 \mathrm{~h}$, these ridges totally disappear and the gel surface goes back to completely flat. (Note: By an analogy with the leveling of a thin liquid film [13], the typical relaxation time of the ridge can be estimated of order $\eta R^{4} / \gamma_{s} H^{3}$, where $\eta$ is the gel viscosity, $\gamma_{s}$ is gel surface tension, $R$ is droplet radius, and $H$ is average gel thickness. Substituting $R, H=1 \mathrm{~mm}, \eta=2 \times 10^{5} \mathrm{Pas}$ (measured by rheometry at low frequencies), $\gamma_{s}=30 \mathrm{mN} / \mathrm{m}$, this is ca. $6000 \mathrm{~s}$, which is close to the relaxation time $2-3$ h observed here.) 
From the sequential pictures, the radius $R$ and contact angle $\theta$ of the droplet are measured as a function of time and are plotted in fig. 3. The data on gels of 2 different SBS concentrations are shown: $c_{S B S}=10 \%$ for (a) and (b), $c_{S B S}=25 \%$ for (c) and (d). The inflation rate is fixed at $q=20 \mu \mathrm{l} / \mathrm{min}$.

The plots in fig. 3 (a) and (b) clearly show that on a gel of $c_{S B S}=10 \%$, the droplet contact line exhibits two different motions. At an early stage of the inflation process $(t<160 \mathrm{sec})$ while the droplet radius $R$ is still sufficiently small $(R<2 \mathrm{~mm})$, the contact line advances continuously with a nearly constant contact angle $\theta_{a} \approx 100^{\circ}$. As $R$ becomes large at a later stage $(t>160 \mathrm{sec}, R>2 \mathrm{~mm})$, the contact line starts the stick-slip motion, i.e., the droplet radius $R$ stays the same value during a certain moment then suddenly increases. Looking at the contact angle, once the contact line sticks, $\theta$ starts to increase from $\theta_{a}$. As $\theta$ reaches to a critical value $\theta_{b} \approx 110^{\circ}$, the contact line slips forward and $\theta$ goes back to the value $\theta_{a}$.

On the other hand, on a rigid gel of higher polymer concentration $c_{S B S}=25 \%$ (fig. 3 (c) and $(\mathrm{d})$ ), the stick-slip motion of the contact line is not observed during the whole inflation process. The droplet contact line always advances continuously with a constant contact angle close to $\theta_{a}$.

\section{B. Dependence on $q$ and $R$ : Three regimes of contact line motion}

For a quantitative analysis, inflating experiments were conducted at various inflating rates $q$. Figure 4 shows the plots of the droplet radius and contact angle against time on gels of $c_{S B S}=10 \%$ at 3 different inflation rates: (a) $q=200 \mu \mathrm{l} / \mathrm{min}$, (b) $20 \mu \mathrm{l} / \mathrm{min}$, and (c) $2 \mu \mathrm{l} / \mathrm{min}$. Since the total inflation time varies largely by $q$, for the comparison of each data, the droplet radius $R$ and time $t$ are normalized by the referential values $R_{r}$ and $t_{r}$. Here $R_{r}$ is arbitrary selected as $4 \mathrm{~mm}$, and $t_{r}$ corresponds the time at which the droplet radius reaches to $R_{r}$ (In our experiment, the typical value of $t_{r}$ is $70 \pm 5 \mathrm{~s}$ for $q=200 \mu \mathrm{l} / \mathrm{min}$, $700 \pm 50 \mathrm{~s}$ for $q=20 \mu \mathrm{l} / \mathrm{min}$, and $6000 \pm 300 \mathrm{~s}$ for $q=2 \mu \mathrm{l} / \mathrm{min})$.

Depending on the inflating rate $q$, the droplet contact line remarkably changes its behavior, which also varies the pattern remaining on the gel surface after the inflation process.

- At a high inflation rate $(q=200 \mu \mathrm{l} / \mathrm{min})$, the contact line advances continuously with a constant contact angle $\theta_{a} \approx 100^{\circ}$ during the whole inflation process (fig. 4 (a)). 
When the droplet is removed after the inflation, the gel surface is flat and no clear trace is observed.

- At an intermediate inflation rate $(q=20 \mu \mathrm{l} / \mathrm{min})$, the contact line initially exhibits the continuous advancing motion. As the droplet radius reaches a critical value $(R \approx 2.2$ $\mathrm{mm}$ ), the contact line starts the stick-slip motion (fig. 4 (b)). $\theta$ is varying between $\theta_{a} \approx 100^{\circ}$ and $\theta_{b} \approx 110^{\circ}$. After the inflation process, multiple circular ridges remain on the gel surface.

- At a low inflation rate $(q=2 \mu \mathrm{l} / \mathrm{min})$, the contact line exhibits the stick-slip motion even at a very early stage of the inflation process. As the droplet radius increases, now the contact line stops the stick-slip motion and starts to advance continuously again (fig. $4(\mathrm{c})$ ). At this moment, the contact angle $\theta$ also stops oscillating between $\theta_{a}$ and $\theta_{b}$, and keeps almost the same value $\theta_{a}$. After the inflation process, the droplet leaves single large lens-like dimple instead of the multiple circular ridges. (Note: Rigorously, $\theta_{a}$ is not constant in the continuous motion and it slightly decreases with the increase of the droplet radius. This means that $\theta_{a}$ is not really a contact angle in a quasi-static limit but rather a value of dynamic contact angle. Nevertheless, as the change of $\theta$ is sufficiently small, here we treat $\theta_{a}$ as a constant of order $100^{\circ}$.)

In order to check that the observed contact line behaviors are peculiar on the SBSparaffin gel substrates, we conducted a supplemental experiment by inflating water droplets on chemically crosslinked silicone elastomer (Sylgard 184; Dow Corning; USA) of Young module $\approx 10 \mathrm{kPa}$. It was observed that on an elastic silicone elastomer, the droplet contact line always advances smoothly with a constant contact angle during the whole inflation process irrespective of the inflation rate $(q=2 \mu \mathrm{l} / \mathrm{min}, 20 \mu \mathrm{l} / \mathrm{min}, 200 \mu \mathrm{l} / \mathrm{min})$.

From the multiple measurements of $R$ and $\theta$ at various inflation rates $q$, we explored which motion the contact line exhibits as a function of $R$, and plotted them in the diagram shown in fig. 5. It is clearly seen that depending on $q$ and $R$, there are three different regimes of the contact line motion:

(i) The smooth advancing regime at high $q$ and small $R$.

(ii) The stick-slip regime at intermediate $q$ and $R$.

(iii) The second smooth advancing regime at low $q$ and large $R$. 


\section{Local deformation of gel surface at a contact line}

To explore the local deformation of the gel surface at the droplet contact line, a sessile droplet of volume $7.5 \mu \mathrm{l}$ was placed on a SBS-paraffin gel and the surface profile close to the contact line was measured by an optical profiler. Figure 6 (a) shows the surface profiles of two gels $\left(c_{S B S}=10 \%\right.$ and $\left.16 \%\right)$ measured shortly after the placement of the droplet $(t=2$ min), and (b) shows the time dependence of the surface profile $\left(c_{S B S}=10 \%, t=2,6,10\right.$ $\min )$.

On both gels, the gel surface is pulled up and a ridge is formed close to the contact line. Since SBS-paraffin gel is hydrophobic and there is no volume exchange between the droplet and gel, the surface ridge is considered as a consequence of the balance between the vertical component of liquid surface tension and the elastic resistance of the gel in analogy with the problem of liquid droplets on elastomer [24-26]. This can be confirmed by fitting the profile in fig. 6 (a) with the theoretical curve of elastic surface ridge due to the capillary force proposed in ref. $[24,25]$

$$
h(x) \approx \frac{h_{0}}{2 \pi} \ln \left(\frac{d}{x}\right) .
$$

The fitting parameter $h_{0}$ corresponds $\gamma \sin \theta / G$ where $G$ is a shear modulus of the gel, $\theta$ is a droplet contact angle, and $\gamma$ is the surface tension of water $\approx 72 \mathrm{mN} / \mathrm{m}$, and the parameter $d$ indicates the macroscopic cutoff length of the surface ridge. (Note: In this model, $h(x)$ as well as $h^{\prime}(x)$ diverges at the contact line $x=0$. More elaborated models were developed recently [27-29] by introducing a microscopic cut-off length due to the substrate surface tension. We will discuss later in section IV-C, but on a scale larger than our resolution $\geq 10$ $\mu \mathrm{m}$, these models also give a similar logarithmic profile. Therefore, we use the model of ref. $[24,25]$ for simplicity.)

Both experimental data well fit with the theoretical curves, giving the fitting parameters $h_{0}=14.4 \mu \mathrm{m}$ and $d=840 \mu \mathrm{m}$ for $c_{S B S}=10 \%$, and $h_{0}=1.64 \mu \mathrm{m}$ and $d=170 \mu \mathrm{m}$ for $c_{S B S}=16 \%$. The typical lengths of $h_{0}$ and $d$ are thus sufficiently smaller than the gel thickness. From the obtained value of $h_{0}$, the shear moduli of gels $G$ were calculated as 5.1 $\mathrm{kPa}$ for $c_{S B S}=10 \%$ and $42 \mathrm{kPa}$ for $c_{S B S}=16 \%$, which are close to the values measured by rheometry.

Meanwhile, the profile of the surface ridge is not persistent as it is shown in fig. 6 (b). If the contact line stays at the same position on the SBS-paraffin gel, the foot of the surface 
ridge gradually expands outward, and the profile of the ridge becomes less steep.

\section{DISCUSSION}

In this chapter, we will discuss why the contact line exhibits three different motions (i)(iii) as seen in fig. 5, and propose simple geometric considerations in the stick-slip regime (ii).

\section{A. Three contact line motions: separation by the characteristic frequency}

Considering that both the parameters $q$ and $R$ affect the velocity of the advancing contact line, here we estimate the apparent mean contact line velocity $v$. $v$ is determined as the velocity of the contact line supposing that the droplet always keeps the contact line advancing continuously with a constant contact angle $\theta_{a}$ at given $q$ and $R$ (Note that $v$ is not the actual contact line velocity in the stick-slip regime.). From the change of the droplet volume

$$
V_{\text {drop }}=\frac{\pi R^{3}}{3}\left(2-3 \cos \theta_{a}\right)
$$

by the constant volume rate $q$

$$
d V_{d r o p}=q d t
$$

$v$ is calculated as

$$
v=\frac{d R}{d t} \approx \frac{q}{2 \pi R^{2}}
$$

as the advancing angle $\theta_{a}$ is close to $90^{\circ}$ in our experiment.

In order to compare the contact line behavior with the rheological properties of the gel, we further estimate the characteristic frequency of the gel surface deformation at the moving contact line. Looking back at fig. 6, the surface deformation is maximum at the contact line and it goes to zero at distances larger than the droplet radius $R$. Supposing the situation that the droplet is moving forward or backward around a certain point of the gel, the surface on that point rises as the contact line approaches, then falls as the contact line goes away. The typical period of the surface rise and fall $\tau$ is thus given by dividing the scale of the surface deformation $R$ by the contact line velocity $v$. Its inverse is the characteristic frequency of the surface deformation,

$$
f=1 / \tau=v / R
$$


Of the data points in fig. 5, we calculated the characteristic frequency and re-plotted the diagram as a function of $f$ in fig. 7 (a). It is clearly observed that the three regimes of the contact line motions (i)-(iii) are separated against $f$, giving two critical frequencies which characterize the transition between the different regimes: $f_{c 1}$ separates between the (i) continuous advancing motion at a high frequency and (ii) stick-slip regime, and $f_{c 2}$ separates between the (ii) stick-slip and (iii) continuous advancing at a low frequency. Comparing this diagram with the rheology of the gel in fig. 1, it is also seen that the crossover frequency $f_{\text {cross }}$ where the two moduli correspond with each other $G^{\prime \prime} / G^{\prime}=1$ is between these two critical frequencies $f_{c 1}$ and $f_{c 2}$.

To explore if the critical frequencies $f_{c 1}$ and $f_{c 2}$ depend on the property of gel, we conducted the inflation experiments of water droplets on gels of various SBS concentrations. Figure 7 (b) shows a plot of $f_{c 1}$ and $f_{c 2}$ as a function of $c_{S B S}$. For reference, the crossover frequency of the gel $f_{\text {cross }}$ obtained by rheometry is also plotted. It is observed that both the critical frequencies $f_{c 1}$ and $f_{c 2}$ decrease with the increase of $c_{S B S}$, indicating that $f_{c 1}$ and $f_{c 2}$ have a dependence on $f_{\text {cross }}$. On a rigid gel of high SBS concentration $\left(c_{S B S}=25 \%\right)$ having a low $f_{\text {cross }}$, the regime (i) becomes prominent in a wide range of $f$ and the value of $f_{c 1}$ becomes considerably low $\left(\approx 10^{-4} 1 / \mathrm{s}\right)$. On the contrary, on a soft gel of low SBS concentration $\left(c_{S B S}=8 \%\right)$ having a high $f_{\text {cross }}$, both $f_{c 1}$ and $f_{c 2}$ drastically shifted to higher values $\left(f_{c 1} \approx 2.2 \times 10^{-2} 1 / \mathrm{s}\right.$ and $\left.f_{c 2} \approx 2.0 \times 10^{-3} 1 / \mathrm{s}\right)$, and the contact line mostly takes the regimes (ii) and (iii).

\section{B. Mechanism of the contact line motion transition}

Based on the results in the previous sections, we propose a mechanism for the transition between the three contact line behaviors.

As discussed in sec. III-C, initially the surface deformation at the contact line is caused by the balance between the surface tension of the liquid and elastic resistance of the gel. However, as the SBS-paraffin gel is visco-elastic, when the contact line stays close to its initial position, the elastic resistance of the gel gradually relaxes and the gel creeps to evolve a surface as shown in fig. 6 (b). After a sufficiently long time, the surface ridge changes its shape to a gradual surface rise like as the capillary ridge formed at the contact line of liquid on a surface of another liquid [30]. 
Considering the change of the surface deformation due to the visco-elastic nature of the gel $[31,32]$, the transition of the contact line motions can be understood in terms of the characteristic frequency $f$ of the surface deformation. Figure 8 illustrates schematically the mechanism of the three contact line motions (i)-(iii).

- Regime (i): When the contact line advances quickly and the characteristic frequency is high $\left(f \gg f_{\text {cross }}\right)$, the gel substrate responds as an elastic solid to the motion of the contact line. In this situation, the surface deformation at the contact line is an elastic ridge pulled by the surface tension of liquid. Therefore, the elastic ridge can always follow the motion of the contact line at which the force is locally applied, resulting in the continuous advancing motion (In SBS-Paraffin gel, the elastic deformation can propagate up to a velocity of the elastic wave $\sqrt{G / \rho} \sim 1 \mathrm{~m} / \mathrm{s}$ [33], which is much higher than the velocity in our experiment).

- Regime (ii): As the velocity of the contact line decreases and $f$ comes close to the crossover frequency $\left(f \approx f_{\text {cross }}\right)$, now the response of the gel surface is not purely elastic. Until the contact line advances over a distance proportional to $R$, the elastic resistance of the gel already starts to relax and a part of the surface deformation becomes irreversible. The surface ridge at the contact line behaves something like a "surface defect" which pins the contact line. The contact line has to jump over this defect for advancing, resulting in the stick-slip motion.

- Regime (iii): In the other extreme situation, when the contact line advances slowly and $f$ is sufficiently low $\left(f \ll f_{\text {cross }}\right)$, the deformation of the gel surface totally relaxes until the contact line moves over a distance of order $R$. In this case, the gel substrate behaves nearly like a sheet of very viscous liquid with respect to the contact line motion. As the contact line moves, a capillary flow is driven below the gel surface due to the position shift of the surface force. The surface deformation is transported forward by this capillary flow, following the motion of the contact line. Therefore, the contact line exhibits a continuous advancing motion. This is also consistent with the formation of the lens-like dimple shown in fig. 4 (c"). (Note: At an early stage in fig. 4 (c), there are two stick-slip motions. However, as the total inflation time $(\approx 6000 \mathrm{~s})$ is sufficiently long, the surface ridges formed at these two stick points already relax.) Now looking back at the regime (ii) in comparison with the regime (iii), the onset of 
the stick-slip motion $f_{c 2}$ can be interpreted as the frequency at which the flow inside the gel cannot follow the contact line as the elastic stress oppose the substrate flow.

The aforementioned mechanism predicts that the rheological property of the gel, especially the crossover frequency $f_{\text {cross }}$ largely affects the transition of the contact line motions (i)-(iii). This is also consistent with our experimental results. As we have observed in fig. 7 (b), both the critical frequencies $f_{c 1}$ and $f_{c 2}$ drastically change depending on the $f_{\text {cross }}$. The contact line mostly exhibits the regime (i) on a rigid gel of low $f_{\text {cross }}$, while on a soft gel of high $f_{\text {cross }}$, the regimes (ii) and (iii) become more prominent.

\section{Simple geometric considerations in the stick-slip regime}

In this section, we propose geometric considerations in the stick-slip regime (ii) that allows to predict the link between jumps of the radius and jumps of the apparent contact angle. The schematic of our model is depicted in fig. 9 (a). Inspired by the model of contact angle hysteresis on elastomer by Extrand and Kumagai [34], here we are considering a simple geometric relation of droplet shapes just before and after one slip motion. The variables $R_{b}, \theta_{b}$ and $R_{a}, \theta_{a}$ are the droplet radii and apparent contact angles before and after the contact line slips on the surface ridge, and $\phi$ indicates the angle of the local slope of surface ridge.

Considering that the droplet volume $V$ is conserved between before and after the slip, the relations of droplet radii and contact angles are expressed as

$$
V=\frac{\pi R_{b}^{3}}{3}\left(2-3 \cos \theta_{b}\right)=\frac{\pi R_{a}^{3}}{3}\left(2-3 \cos \theta_{a}\right) .
$$

We suppose that $\theta_{a}$ is an advancing contact angle on an undeformed surface, and that the difference between $\theta_{b}$ and $\theta_{a}$ is essentially due to the local surface slope. To advance on a inclined surface, the contact line has to take an advancing angle equal to

$$
\theta_{b}=\phi+\theta_{a}
$$

As $\theta_{a}$ is close to $90^{\circ}$ in our situation, the equations (6) and (7) are re-written as a function of the ratio of two radii

$$
\begin{aligned}
\left(\frac{R_{a}}{R_{b}}\right)^{3} & =\frac{2-3 \cos \left(\theta_{a}+\phi\right)}{2-3 \cos \theta_{a}} \\
& \approx 1+\frac{3}{2}\left[\cos \theta_{a}-\cos \left(\theta_{a}+\phi\right)\right] .
\end{aligned}
$$


With an approximation that $\phi$ is sufficiently small, the ratio $R_{a} / R_{b}$ relates to $\phi$ as shown in the following expression

$$
\begin{aligned}
\frac{R_{a}}{R_{b}} & \approx 1+\frac{1}{2}\left[\cos \theta_{a}-\cos \left(\theta_{a}+\phi\right)\right], \\
& \approx 1+\frac{\phi}{2}
\end{aligned}
$$

This equation links the ratio of successive droplet radii to the local surface slope $\phi$

$$
\phi=\frac{2\left(R_{a}-R_{b}\right)}{R_{b}}=2 \frac{\Delta R}{R_{b}} .
$$

In order to check this model, we used the experimental data of $c_{S B S}=10 \%$ in the stick-slip regime (ii) and calculated the quantity $2 \Delta R / R_{b}$ for various stick-slip motions. We compared it to the value of $\phi=\theta_{b}-\theta_{a}$ extracted from the direct measurement of the contact angle $\theta$. Figure 9 (b) shows the plot of $2 \Delta R / R_{b}$ and of $\phi$ as a function of the characteristic frequency $f$. Both quantities are noted nearly equal, which indicates that our model has captured successfully the essential features.

Alternatively, the local slope of the gel surface close to the contact line was measured from the profile curve in fig. 6 (a). The experimental data gives the slope angle ca. $6.5^{\circ}$ at $x=10 \mu \mathrm{m}$, which is close to the value $10^{\circ}$ obtained here.

Finally, let us close this section by qualitatively discussing the fact that $\phi$ remains locked at the value $10^{\circ}$ independently on $f$. These results are bit surprising because in available modeling in eq. (1) $[24,25]$, the local slope of the surface ridge $h^{\prime}(x)$ depends on the elastic modulus $G$ and thus, here, indirectly on $f$, and even diverges near $x=0$.

All seems here to happen as if $\phi$ is selected by some truncation of this profile at a small scale eliminating the contribution of $G$. In recent studies of wetting on elastomer, this truncation of the surface profile at the contact line was proposed to be ruled by the surface tension of the substrate $\gamma_{s}$ [27-29], as observed experimentally by Jerison et al. [27]. In short, for a scale smaller than the elasto-capillary length $l_{e}=\gamma_{s} / G$, which plays a role of cut-off, the surface profile is locally determined by the balance of three interfacial tensions and does not depend on the substrate elasticity. For a paraffin gel, the typical value of $\gamma_{s}$ is $30 \mathrm{mN} / \mathrm{m}[35,36]$, giving the value of $l_{e} \approx 6 \mu \mathrm{m}$ for $G=5 \mathrm{kPa}$. This value is smaller than our experimental resolution, but it has the right order of magnitude. In addition to this putative cut-off length, when we look at fig. 6 (b), the profile of the surface in the vicinity of 
the contact line $(10 \mu \mathrm{m} \leq x \leq 50 \mu \mathrm{m})$ does not evolve significantly within our time window. From these remarks, it is quite natural that $\phi$ does not depend on $f$.

Although the models for the wetting on elastomer can not be applied directly here as the paraffin gel is visco-elastic and the three interfacial tensions cannot be balanced in our situation $\left(\gamma>2 \gamma_{s}\right)$, our results suggest that one of their main prediction, i.e. the relevance of elasto-capillary length at small scale, could also hold in this very complex problem. Further studies will be expected to conclude this proposition definitely.

\section{CONCLUSION}

In this paper, we have studied the dynamics of water sessile droplets advancing on viscoelastic SBS-paraffin gel substrates. We have observed that the droplet contact line exhibits three different regimes of motions, and their transition is characterized by a frequency of the surface deformation $f$ determined by the apparent contact line velocity $v$ and droplet radius $R$ as $f=v / R$. At high $f$ with respect to the cross over frequency of gel $f_{\text {cross }}$, the contact line moves continuously with a constant contact angle. As the contact line slows down and $f$ approaches to $f_{\text {cross }}$, the contact line starts the stick-slip motion: the contact line is pinned at a certain position, then suddenly slips forward. In this stick-slip regime, the contact line leaves circular stripe ridges on the gel surface. At $f$ sufficiently lower than $f_{\text {cross }}$, the contact line stops stick-slip and advances continuously again.

We have conjectured that the observed transitions of the contact line motions (continuous - stick-slip - continuous) are the consequence of the rheological property of the gel affecting the dynamics of contact line. Depending on the frequency, the behavior of the liquid contact line on gels shows both aspects of wetting on elastic solids and on viscous liquid sheets. At an intermediate frequency where the gel behaves neither solid nor liquid, the stick-slip motion appears. Simple geometrical considerations in the stick-slip regime have also been proposed.

\section{Acknowledgement}

The authors gratefully thank G. Ducouret and A. Chateauminois for their various supports for the rheometry and for surface profile measurement. T.K. is supported by the JSPS Postdoctoral Fellowship for Research Abroad (No. 22-120) from the MEXT of Japan. 
[1] A. Suzuki and T. Tanaka, Nature, 1990, 346, 345-347.

[2] M. Doi, J. Phys. Soc. Jpn., 2009, 78, 052001:1-19.

[3] X. Hu, L. Hao, H. Wang, X. Yang, G. Zhang, G. Wang and X. Zhang, Int. J. Pol. Sci., 2011, 2011, 814163:1-9

[4] H. Bong, W. H. Lee, D. Y. Lee, B. J. Kim, J. H. Cho and K. Cho, Appl. Phys. Lett., 2010, 96, 192115:1-3.

[5] T. Baumberger, C. Caroli and D. Martina, Nature Mat., 2006, 5, 552-555.

[6] M. Morishita, M. Kobayashi, T. Yamaguchi and M. Doi, J. Phys. Condens. Matter, 2010, 22, 365104:1-6.

[7] D. Kaneko, T. Tada, T. Kurokawa, J. P. Gong and Y. Osada, Adv. Mater., 2005, 17, 535-538.

[8] D. Szabó, S. Akiyoshi, T. Matsunaga, J. P. Gong and Y. Osada, J. Chem. Phys., 2000, 113, 8253-8259.

[9] Y. Nonomura, Y. Morita, T. Hikima, E. Seino, S. Chida and H. Mayama, Langmuir, 2010, 26, $16150-16154$.

[10] J. M. Lakkis, Encapsulation and Controlled Release Technologies in Food Systems, WileyBlackwell, 2007.

[11] R. B. Bhatia, C. J. Brinker, A. K. Gupta and A. K. Singh, Chem. Mater. 2000, 12, 2434-2441.

[12] M. Banaha, A. Daerr and L. Limat, Eur. Phys. J. Special Topics, 2009, 166, 185-188.

[13] P. G. de Gennes, F. B. Wyart and D. Quere, Capillarity and Wetting Phenomena, Springer, 2003.

[14] D. Bonn, J. Eggers, J. Indekeu, J. Meunier and E. Rolley, Rev. Mod. Phys. 2009, 81, 739-805.

[15] M. E. R. Shanahan and P. G. de Gennes, C. R. Acad. Sci. Paris, 1986, 302, Ser. II, 517.

[16] A. Carré and M. E. R. Shanahan, Langmuir, 2001, 17, 2982-2985.

[17] B. Roman and J. Bico, J. Phys.: Condens. Matter, 2010, 22, 493101:1-16.

[18] A. Aradian, E. Raphael and P.G. de gennes, Eur. Phys. J. E: Soft Matter Biol. Phys, 2000, 2, 367-376.

[19] L. Bacri and F. B. Wyart, Eur. Phys. J. E: Soft Matter Biol. Phys, 2000, 3, 87-97.

[20] D. P. Holmes, M. Roché, T. Sinha and H. A. Stone, Soft Matter, 2011, 7, 5188-5193.

[21] T. Kajiya, A. Daerr, T. Narita, L. Royon, F. Lequeux and L. Limat, Soft Matter, 2011, 7, 
11425-11432.

[22] G. Pu and S. J. Severtson, Langmuir, 2008, 24, 4685-4692.

[23] G. Pu, J. Ai and S. J. Severtson, Langmuir, 2010, 26, 12696-12702.

[24] M. E. R. Shanahan, J. Phys. D: Appl. Phys., 1987, 20, 945-950.

[25] A. Carré, J. C. Gastel and M.E.R. Shanahan, Nature, 1996, 379, 432-434.

[26] R. P. Cámara, G. K. Auernhammer, K. Koynov, S. Lorenzoni, R. Raiteri and E. Bonaccurso, Soft Matter, 2009, 5, 3611-3617.

[27] E. R. Jerison, Y. Xu, L. A. Wilen and E. R. Dufresne, Phys. Rev. Lett., 2011, 106, 186103:1-4.

[28] A. Marchand, S. Das, J. H. Snoeijer and B. Andreotti, Phys. Rev. Lett, 2012, 108, 094301:1-4.

[29] L. Limat, Straight contact lines on a soft solid, unpublished work.

[30] J. C. Burton, F. M. Huisman, P. Alison, P. Rogerson and P. Taborek, Langmuir, 2010, 26, 15316-15324.

[31] D. Long, A. Ajdari and L. Leibler, Langmuir, 1996, 12, 1675-1680.

[32] D. Long, A. Ajdari and L. Leibler, Langmuir, 1996, 12, 5221-5230.

[33] L. D. Landau and E. M. Lifshitz, Theory of Elasticity, Butterworth Heinemann, 3rd edn., 1986.

[34] C. W. Extrand and Y. Kumagai, J. Colloid Interface Sci., 1996, 184, 191-200.

[35] A. J. Queimada, I. M. Marrucho and J. A. P. Coutinho, Fluid Phase Equilib., 2001, 183-184, 229-238.

[36] M. Talreja, I. Kusaka and D. L. Tomasko, Fluid Phase Equilib., 2012, 319, 67-76. 


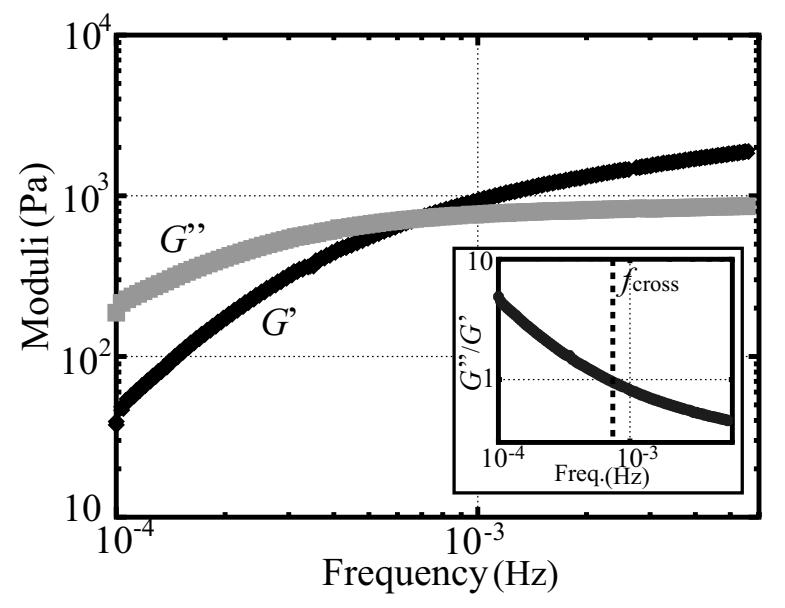

FIG. 1: Storage and loss moduli $G^{\prime}$ and $G^{\prime \prime}$ of the paraffin gel $\left(c_{S B S}=10 \%\right)$ at $20^{\circ} \mathrm{C}$. Phase angle $\tan \delta=G^{\prime \prime} / G^{\prime}$ is shown in the inset.

TABLE I: Typical value of storage modulus $G$ ' at $1 \mathrm{~Hz}$ and crossover frequency $f_{\text {cross }}$ where $G^{\prime \prime} / G^{\prime}=1$ of SBS-Paraffin gel.

\begin{tabular}{|c|c|c|c|c|}
\hline$c_{S B S}(\%)$ & 8 & 10 & 12 & 16 \\
\hline$G^{\prime}(\mathrm{kPa})$ & 2.53 & 5.72 & 13.1 & 19.4 \\
\hline$f_{\text {cross }}(1 / \mathrm{s})$ & $3.3 \times 10^{-3}$ & $7.2 \times 10^{-4}$ & $5.3 \times 10^{-5}$ & $2.6 \times 10^{-6}$ \\
\hline
\end{tabular}




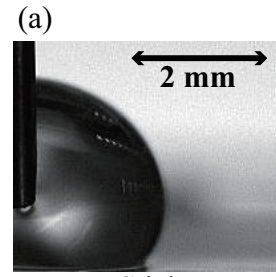

(c)

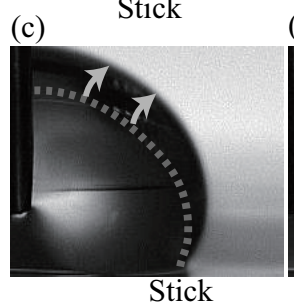

(d)

(b)

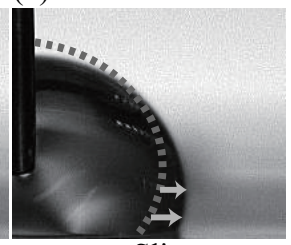

Slip

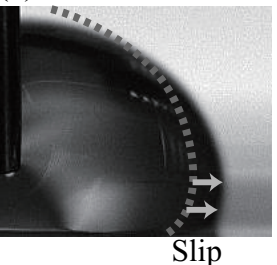

(e)

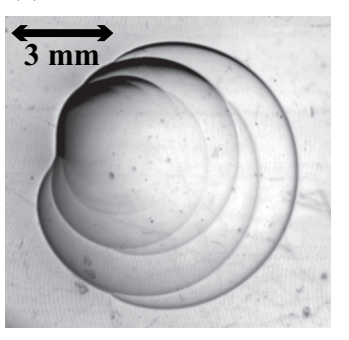

FIG. 2: (a)-(d) Sequential pictures of water droplet being inflated on SBS-paraffin gel $\left(c_{S B S}=10 \%\right)$ at an inflation rate $q=20 \mu \mathrm{l} / \mathrm{min}$. In images (b)-(d), a contour line of the previous droplet shape is drawn as a dotted line. During the inflation process, the contact line shows stick-slip behavior, i.e., the contact is fixed during a certain moment, then quickly advances. (e) A picture of the gel surface taken after the inflation process is finished and the droplet is removed. The droplet leaves multiple circular patterns. 

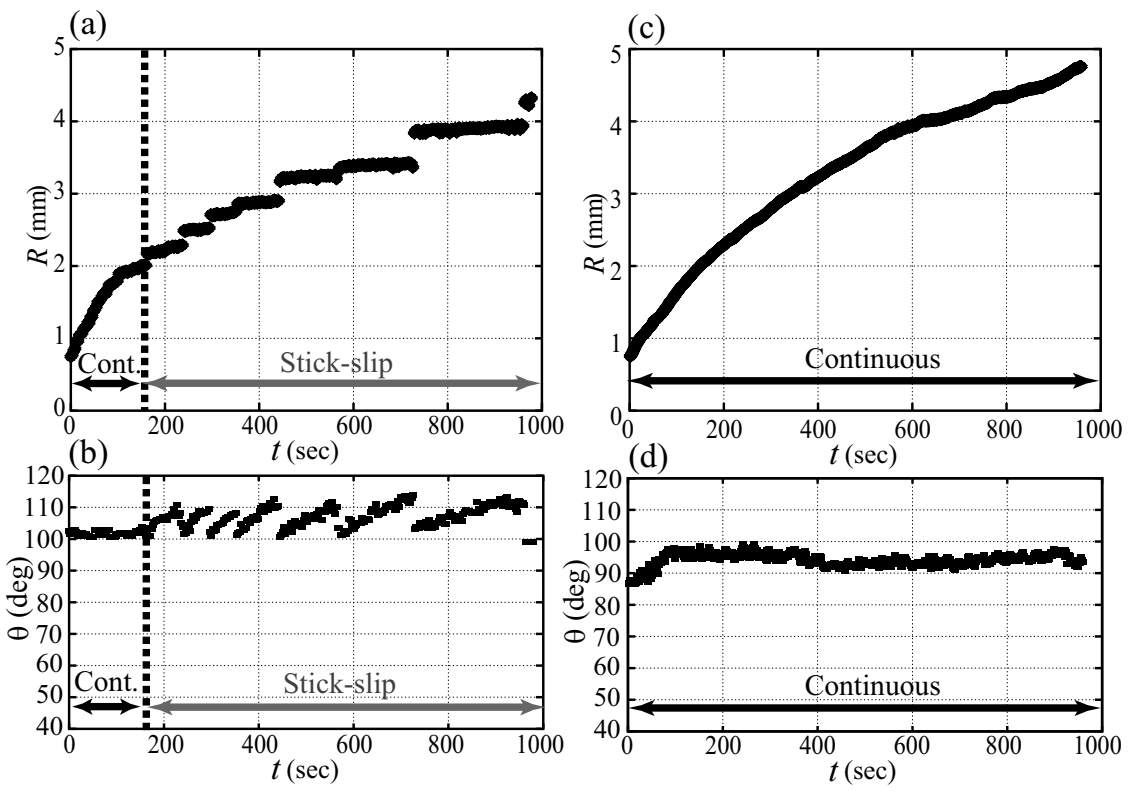

FIG. 3: Plots of the radii and contact angles of droplets against time being inflated on SBS-paraffin gels at an inflation rate $q=20 \mu \mathrm{l} / \mathrm{min}$. Data on gels of two different SBS concentrations are shown. (a) Radius and (b) angle of droplet on a gel of $c_{S B S}=10 \%$. (c) Radius and (d) angle of droplet on a gel of $c_{S B S}=25 \%$. 

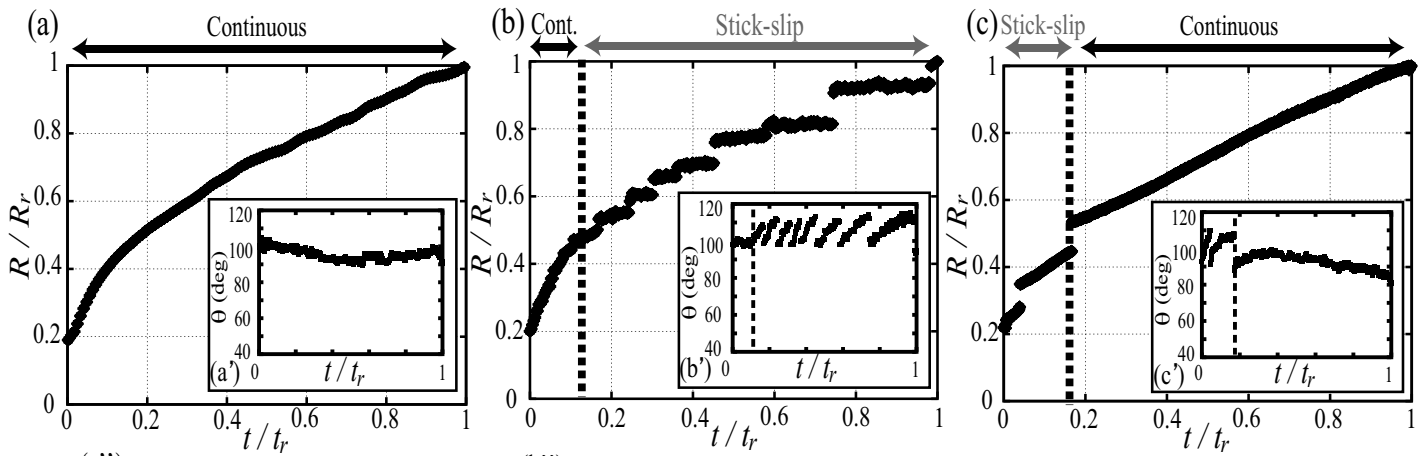

(a")

(b")

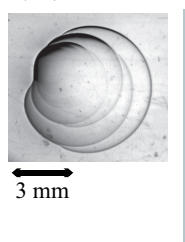

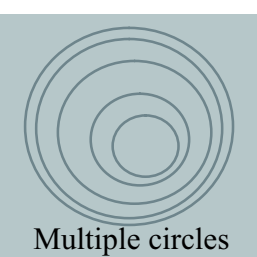

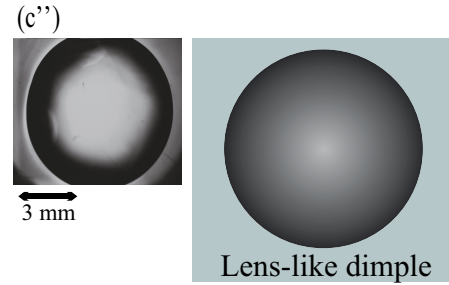

FIG. 4: Plots of the normalized radii of droplets against normalized time being inflated on SBSparaffin gels of $c_{S B S}=10 \%$ at three different inflation rates. For comparison, the radius is normalized by a referential value $R_{r}=4 \mathrm{~mm}$, and the time is normalized by a time $t_{r}$ at which the radius reaches $R_{r}$. The inflation rates are (a) $q=200 \mu \mathrm{l} / \mathrm{min}$, (b) $q=20 \mu \mathrm{l} / \mathrm{min}$, (c) $q=2$ $\mu \mathrm{l} / \mathrm{min}$. The contact angles are also plotted in the insets (a'), (b') and (c'), and the pictures and schematic images of the gel surface after the inflation process are shown in the insets (a"), (b") and $(\mathrm{c} ")$. 


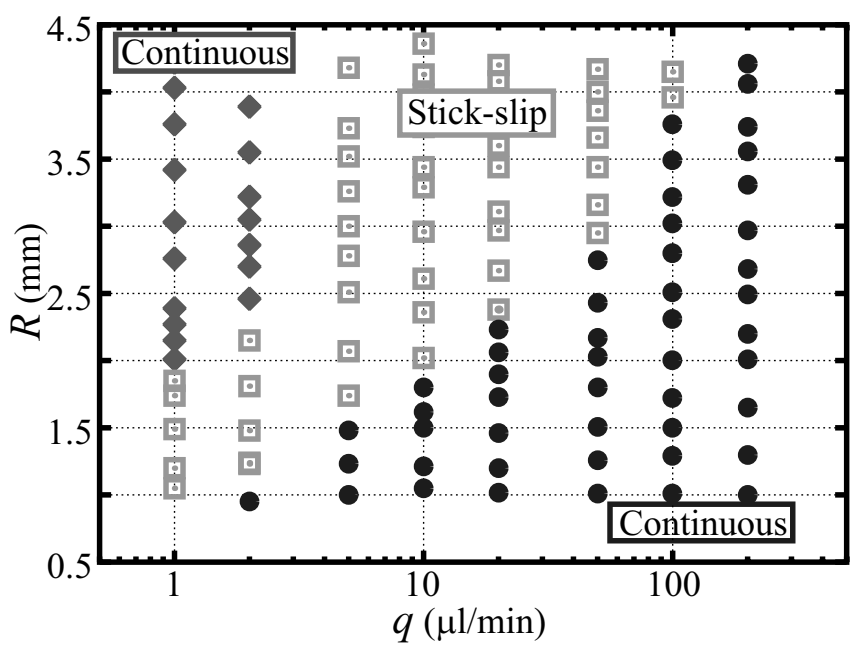

FIG. 5: Diagram of the three different regimes of the contact line motion observed on gels of $c_{S B S}=10 \%$. Data of various droplet radii $R$ and inflation rates $q$ are plotted. The three regimes are distinguished by different symbols, i.e., (i) circle: continuous advancing observed at high $q$ and small $R$, (ii) square: stick-slip motion, (iii) diamond: continuous advancing at low $q$ and large $R$. 
(a)
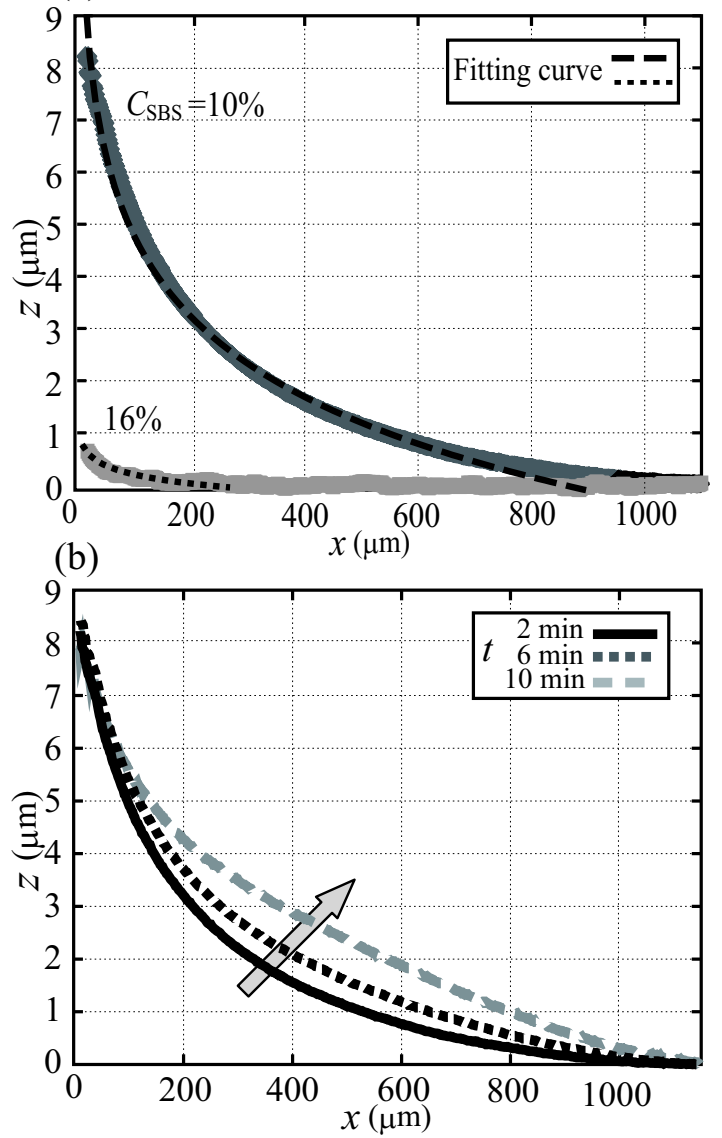

FIG. 6: Cross sections of the gel surface profiles close to the contact line of the water droplet of volume $7.5 \mu \mathrm{l}$. The position $x=0$ indicates the position of the contact line. Note that the profile data were obtained from $x \geq 10 \mu \mathrm{m}$ which is our limit of resolution. (a) Gel surface profiles of 2 different $c_{S B S}(10 \%, 16 \%)$. The profiles were measured 2 minutes after the placement of the droplet. Bold lines indicate the experimental data, and the dashed lines are the fitting curves of the theoretical formula in ref. $[15,16]$. (b) Time evolution of the surface profile on the gel of $c_{S B S}=10 \%$. 

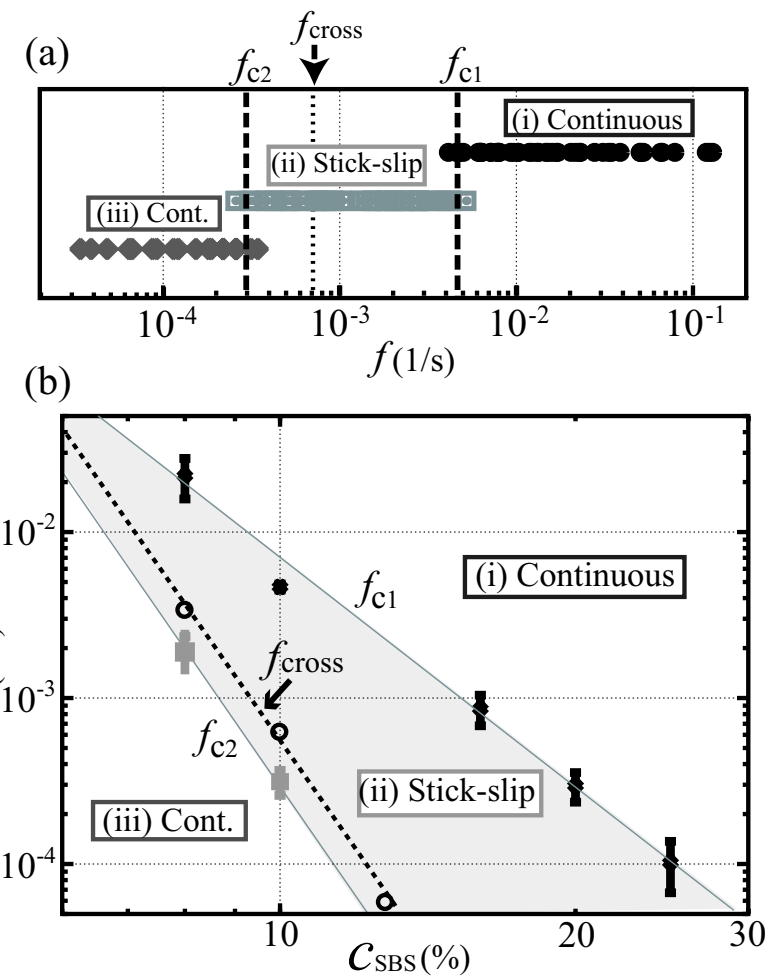

FIG. 7: (a) Replot of the data in fig. 5 against the characteristic frequency $f=q / 2 \pi R^{3}$. The crossover frequency $f_{\text {cross }}$ where $G^{\prime \prime} / G^{\prime}=1$ is marked by an arrow. Two critical frequencies $f_{c 1}$ and $f_{c 2}$ are observed which separate the different regimes. (b) Plot of the two critical frequencies $f_{c 1}$ and $f_{c 2}$ against SBS concentration of gel $c_{S B S}$. The crossover frequencies of gels $f_{\text {cross }}$ are plotted as hollow circles for reference. 
(i) $f \gg f_{\text {cross }}$

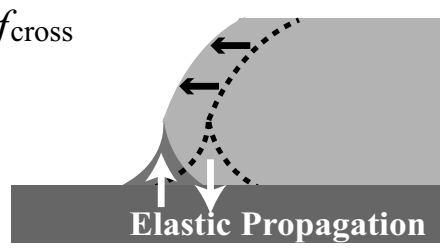

(ii) $f \approx f_{\text {cross }}$

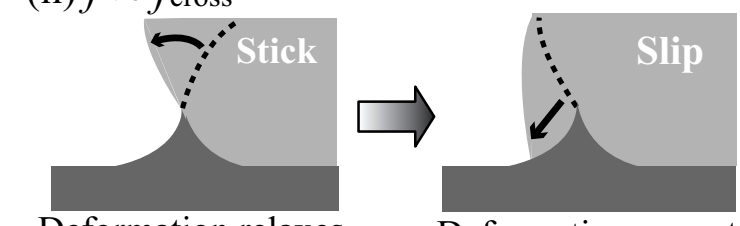

Deformation relaxes Deformation cannot and pins contact line. follow contact line.

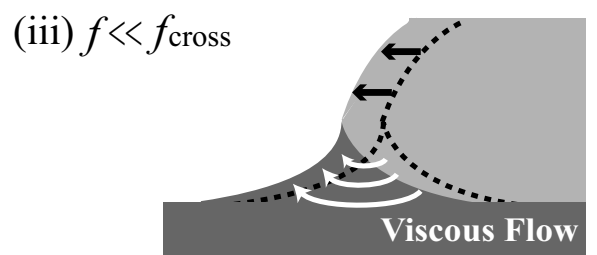

FIG. 8: Behaviors of the contact line and of gel surface deformation at different regimes (i)-(iii). 
(a)

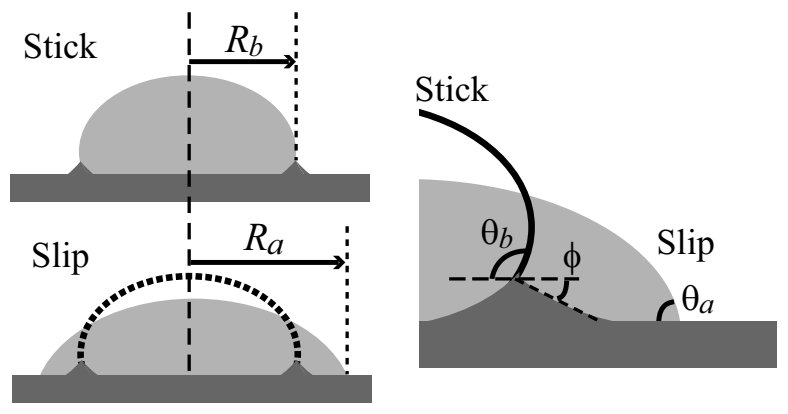

(b)

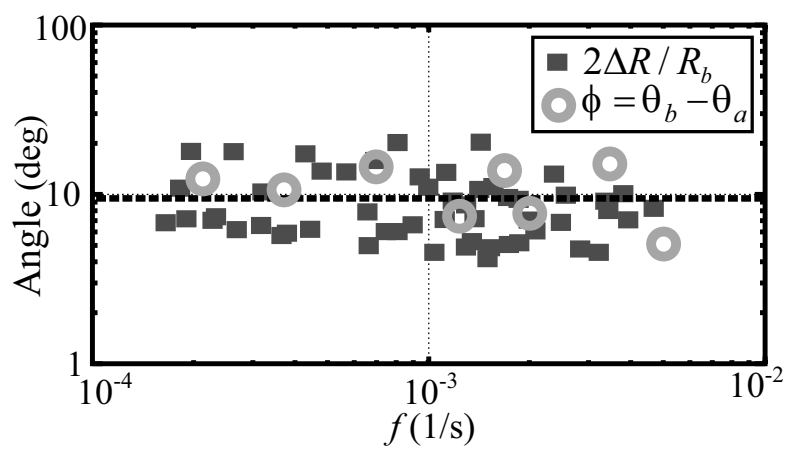

FIG. 9: (a) Parameters for the geometric considerations in the stick-slip regime. $R_{b}, \theta_{b}$ and $R_{a}, \theta_{a}$ are radii and contact angles before and after one slip motion, and $\phi$ indicates the local slope of the surface ridge. (b) Plot of the angle of the local slope of gel surface predicted from the ratio of successive droplet radii $2 \Delta R / R_{b}$ against $f$. For comparison, the value of $\phi=\theta_{b}-\theta_{a}$ directly obtained from the contact angle measurement was plotted as hollow circles. 